\title{
Light-Switchable Polymer Adhesive Based on Photoinduced Reversible Solid-to-Liquid Transitions
}

\author{
Ying Zhou, ${ }^{\dagger, \ddagger, \S}$ Mingsen Chen, ${ }^{\ddagger}$ Qingfu Ban, ${ }^{\ddagger}, \|$ Zhenlin Zhang, ${ }^{\ddagger}$ Shaomin Shuang, ${ }^{\S}$ Kaloian Koynov, ${ }^{\ddagger}$

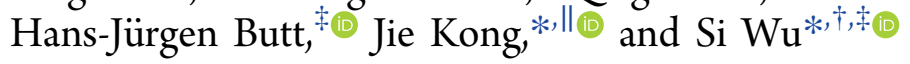

${ }^{\dagger}$ CAS Key Laboratory of Soft Matter Chemistry, Hefei National Laboratory for Physical Sciences at the Microscale, Anhui Key Laboratory of Optoelectronic Science and Technology, Department of Polymer Science and Engineering, University of Science and Technology of China, Hefei 230026, China

${ }^{\ddagger}$ Max Planck Institute for Polymer Research, Ackermannweg 10, 55128 Mainz, Germany

${ }^{\S}$ School of Chemistry and Chemical Engineering, Shanxi University, Taiyuan 030006, China

"MOE Key Lab of Materials Physics and Chemistry in Extraordinary Conditions, Shaanxi Key Lab of Macromolecular Science and Technology, School of Science, Northwestern Polytechnical University, Xi'an 710072, China

\section{Supporting Information}

ABSTRACT: The development of switchable adhesives for reversible bonding and debonding can overcome the problems associated with conventional adhesives in separating, recycling, and repairing glued surfaces. Here, a photoresponsive azobenzene-containing polymer (azopolymer) is developed for photocontrolled adhesion. The azopolymer P1 (poly(6-(4-( $p$-tolyldiazenyl)phenoxy)hexyl acrylate $))$ exhibits photoinduced reversible solid-to-liquid transitions due to trans-cis photoisomerization. Trans P1 is a solid that glues two substrates with a stiffness comparable to that of conventional adhesives. UV light induces trans-to-cis isomerization, liquefies $\mathrm{P} 1$, weakens the adhesion, and facilitates the separation of glued substrates. Conversely, visible light induces cis-to-trans isomerization, solidifies P1, and enhances the adhesion. P1 enables photocontrolled reversible adhesion for various substrates with different wettability, chemical compositions, and surface roughness. P1 can also be implemented in both dry and wet environments. Light can control the adhesion process with high spatiotemporal resolution when using P1 as a switchable adhesive. Photoinduced reversible solid-to-liquid transitions represent a strategy for materials recycling and automated production processes that require reversible bonding and debonding.

A dhesives are materials that bind two separate surfaces together and resist separation. Adhesives are widely used in daily life, from the packing industry to the construction of complex structures such as skyscrapers, furniture, and ships. The development of novel adhesives for new applications (e.g., automated production and controlling the adhesion of biomaterials) is currently an emerging topic of intense investigation. $^{1-7}$ Surfaces glued by conventional adhesives are difficult to separate, making the separation and reuse of the surfaces inconvenient. In particular, some applications require that glued surfaces are easily separable for recycling or repair. Thus, switchable adhesives, which reversibly bond surfaces on demand, are better suited for material separation and recycling, automated production processes, and the repair of joints.

Switchable adhesives are usually based on reversible chemical or physical processes such as supramolecular interactions, ${ }^{8-16}$ reversible reactions, ${ }^{17-21}$ and reversible phase or topology transitions. ${ }^{2-26}$ On one hand, switchable host-guest interactions have been applied to control underwater adhesion. ${ }^{14-16}$ On the other hand, switchable compounds and dynamic materials have been used to control dry adhesion. ${ }^{17-19,27,28}$ Switchable adhesives based on supramolecular interactions usually require grafting supramolecular adhesives on substrates via chemical reactions. Grafting adhesives on substrates make the use of such switchable adhesives inconvenient. Although switchable adhesives based on dynamic carbon frameworks do not need chemical modification of substrates, they only work at high temperatures $\left(70-135{ }^{\circ} \mathrm{C}\right)$ because of their liquid crystal nature in that temperature range. ${ }^{17}$ In comparison, switchable adhesives that work at ambient temperature are more practical. Furthermore, no adhesive, which can reversibly control the adhesion between different substrates and operate in both dry and wet environments, has been demonstrated.

Here, we report on the design of a novel switchable adhesive that works in both dry and wet environments at ambient temperature for different substrates. The switchable adhesive is based on photoinduced reversible solid-to-liquid transitions of

Received: June 19, 2019

Accepted: July 12, 2019

Published: July 22, 2019 
an azopolymer (Figure 1). Some azobenzene compounds show photoinduced reversible solid-to-liquid transitions. ${ }^{19,28-30}$ In

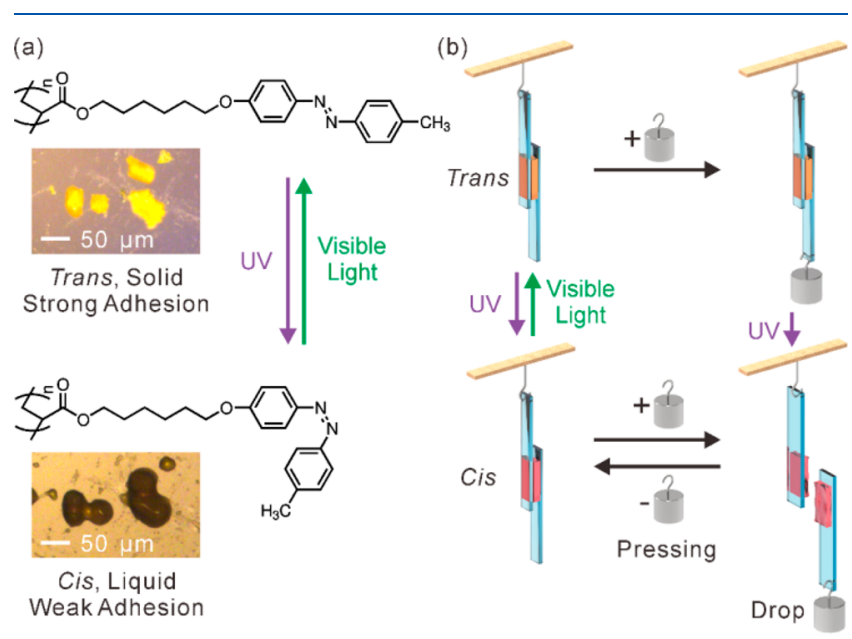

Figure 1. (a) Photoisomerization of the azopolymer P1 induces reversible solid-to-liquid transitions. The insets are optical microscopy images of P1 powders before and after UV irradiation. (b) Schematic illustration of the photoswitchable adhesion of P1-glued substrates. Trans P1 has strong adhesion that can sustain the load. In contrast, cis P1 has weak adhesion, and photoisomerization causes the load to fall.

particular, we recently demonstrated that the poly(6-(4- $(p-$ tolyldiazenyl)phenoxy)hexyl acrylate) (P1) exhibits photoinduced reversible solid-to-liquid transitions due to reversible trans-cis photoisomerization. ${ }^{29}$ Our hypothesis is that P1 in the solid state does not have sufficient chain mobility for separation, while P1 in the liquid state has sufficient chain mobility for separation. In this work, we show that trans P1 is a solid that can firmly bond two substrates. Cis P1 is generated via UV-light-induced trans-to-cis isomerization. Cis P1 is a liquid with weak adhesion that facilitates the separation of the surfaces. Trans P1 can be regenerated by irradiating cis P1 with visible light. Thus, photoinduced reversible solid-to-liquid transitions make the adhesion of P1-glued substrates switchable (Figure $1 \mathrm{~b}$ ). We demonstrate that P1 is a reversible adhesive for different substrates and shows switchable adhesion in both dry and wet environments at ambient temperature.

P1 was synthesized using the synthetic route in the Supporting Information (Figure S1). ${ }^{29}$ Because trans-cis photoisomerization changes glass transition temperatures of azopolymers, ${ }^{29,31}$ P1 can be reversibly liquefied and solidified upon UV and visible light irradiation (Figure 1a). Based on this, we studied photocontrolled adhesion using lap joint shear strength tests (Figure 2a). Two quartz substrates were firmly glued with trans P1 (glued area: $0.375 \mathrm{~cm}^{2}$ ), mounted on a universal testing machine, and pulled until failure. The measured adhesion strength was $\sim 1.02 \mathrm{MPa}$, which is higher than that of small-molecule azobenzene adhesives. ${ }^{\text {9,27 }}$ Irradiation of trans P1 with UV light switched the polymer to the cis form (Figure S2). The adhesion strength of cis P1 was $\sim 0.08 \mathrm{MPa}$, which was only $7.8 \%$ of that of trans P1. The reduction in adhesion occurred because of the photoinduced solid-to-liquid transition of P1 (Figure 1a), which increases the mobility of polymer chains. ${ }^{29}$ Thus, photoisomerization eases the separation of the glued substrates.

The adhesion of trans P1 was so strong that a bottle of water $(\sim 500 \mathrm{~g})$ was sustained using the glued substrates (Figure $2 \mathrm{~b}$ ). UV irradiation weakened the adhesion, and the bottle dropped (a)
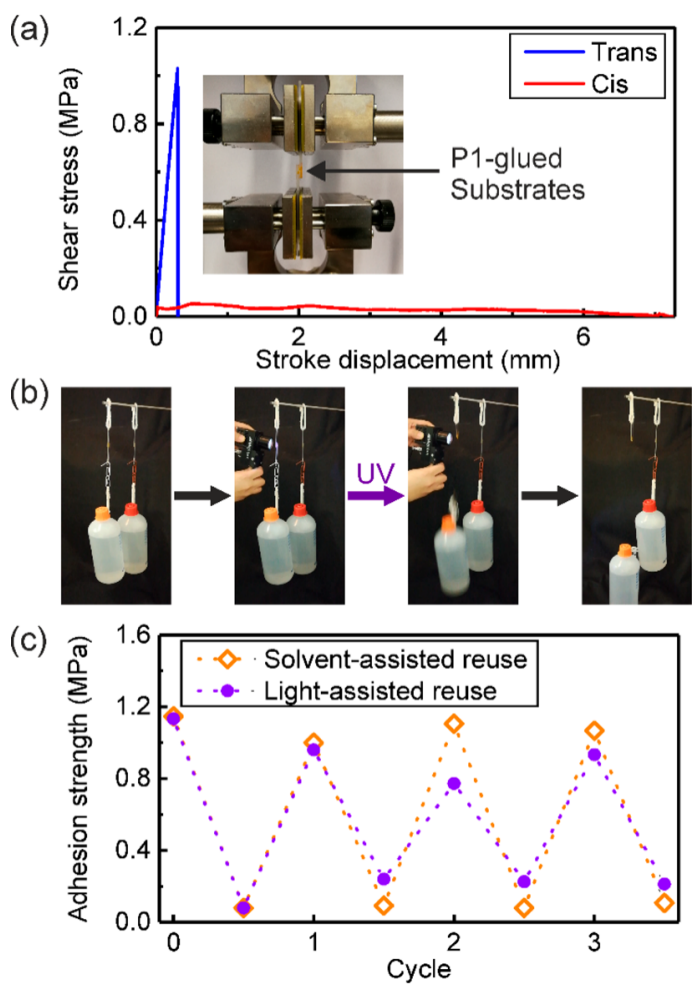

Figure 2. (a) Lap joint shear strength tests with two quartz substrates glued with trans (blue) and cis (red) P1. The inset is a photograph showing P1-glued quartz substrates on the universal testing machine. (b) Snapshots show photocontrolled adhesion (also see Movie S1 in the Supporting Information). Sample on the left: trans-P1-glued quartz substrates holding a bottle of water $(\sim 500 \mathrm{~g})$. The bottle fell after UV irradiation. Sample on the right: a control experiment showing that the P1-glued substrates held the bottle of water when P1 was not irradiated. (c) Light-assisted and solvent-assisted reuse of the adhesive.

after UV irradiation for $\sim 1$ min (see the bottle on the left side of Figure $2 \mathrm{~b}$ and Movie S1). For comparison, P1-glued substrates without UV irradiation (Figure $2 b$, right) sustained a bottle of water with the same weight. UV-vis absorption spectroscopy showed that trans P1 was switched to the cis-P1rich state within a minute. Thus, the adhesion change was in the same time scale of photoisomerization.

Photoinduced reversible solid-to-liquid transitions make P1 a reusable adhesive (Figure $2 \mathrm{c}$ ). To reuse P1 with the assistance of light, P1 was liquefied on two separated quartz substrates via UV-light-induced trans-to-cis isomerization. Then, the substrates were pressed to form a joint. The liquefied P1 wetted the surfaces. Subsequently, P1 was solidified via visible-light-induced cis-to-trans isomerization, which then increased the adhesion. We tested the adhesion of P1 for three photoisomerization cycles (Figure 2c, purple line). The adhesion strength of the reused P1 was more than $68 \%$ of its original strength.

We also demonstrated the reuse of P1 assisted by dichloromethane, which has a low boiling temperature and can be removed easily after use (Figure $2 c$, orange line). Dichloromethane $(10 \mu \mathrm{L})$ was added to the debonded P1 layers on two separated quartz substrates, which increased the mobility of P1 for wetting the substrates. Then, the substrates were pressed to form a joint. P1 was hardened by evaporating dichloromethane in a fume hood for $24 \mathrm{~h}$. The adhesion 
strength of trans P1 was measured after the drying process, whereas the adhesion strength of cis P1 was measured after both the drying process and subsequent UV irradiation. We tested the adhesion of P1 for three cycles (Figure 2c, orange line). The adhesion strength of the reused P1 was more than $87 \%$ of its original strength.

To test whether photoswitchable adhesion is generally applicable to different substrates, we studied different substrates that were glued with P1. First, we used three types of quartz substrates with different wettability (Figure 3a). The first substrate was a native quartz substrate (as purchased) with a static water contact angle of $27 \pm 5^{\circ}$. Superhydrophilic substrates (static water contact angle $<5^{\circ}$ ) were prepared by sonicating quartz in an ethanol solution of $\mathrm{KOH}(4 \mathrm{~mol} / \mathrm{L})$ for $30 \mathrm{~min}$ and washed with water. More hydrophobic substrates (static water contact angle: $89 \pm 5^{\circ}$ ) were prepared by
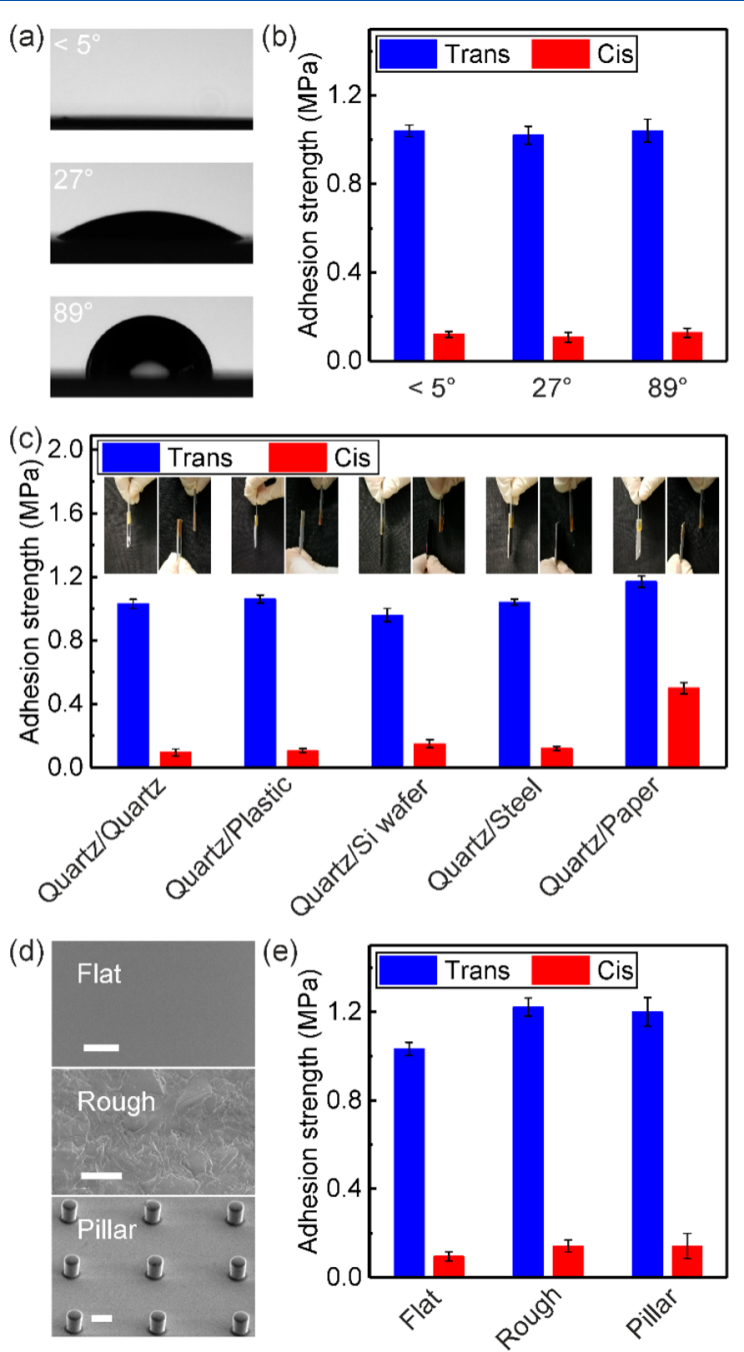

Figure 3. Photoswitchable adhesion of P1 on different substrates. (a) Profiles of a water droplet on substrates with different wettability. (b) Photoswitchable adhesion of P1-glued substrates with different wettability. (c) Photoswitchable adhesion of various P1-glued substrates. The insets are photographs of different substrates glued with trans P1 and separated after trans-cis photoisomerization. (d) SEM images of a flat quartz substrate, a frosted quartz substrate, and a substrate with an array of pillars (scale bars are $5 \mu \mathrm{m}$ ). (e) Photoswitchable adhesion of P1-glued flat quartz substrates, frosted quartz substrates, and pillar-structured substrates. exposing quartz to $1,1,1,3,3,3$-hexamethyldisilazane vapor in a sealed tube for $24 \mathrm{~h}$. Trans P1 firmly bonded all three types of substrates (Figure 3b). UV irradiation induced trans-to-cis isomerization and reduced the adhesion for all three types of substrates. The adhesion strengths of the P1-glued substrates were independent of the wettability. The reason is that the failure was within the P1 layer (see more discussion below).

We also studied the adhesion of different P1-glued substrates such as quartz/quartz, quartz/plastic, quartz/silicon wafer, quartz/steel, and quartz/paper (Figure 3c). The adhesion strengths of all trans-P1-glued substrates were 0.96-1.17 MPa. UV-light-induced trans-to-cis isomerization reduced adhesion. The results showed that the photoswitchable adhesive is applicable to different substrates. The adhesion strengths of the trans and cis P1-glued quartz/paper were the highest for all tested samples. We noticed that the paper had a rough surface (Figure S3), which could enhance adhesion.

To further investigate how surface roughness influences adhesion, we compared P1-glued quartz (flat, RMS roughness of $0.9 \mathrm{~nm}$ ), frosted quartz (rough, RMS roughness of 111.2 $\mathrm{nm}$ ), and pillar-structured substrates (Figure $3 \mathrm{~d}$ and Figure S4). All the studied samples exhibited photoswitchable adhesion (Figure 3e). Trans P1 had stronger adhesion than cis P1. In addition, both P1-glued frosted quartz and pillarstructured substrates had stronger adhesion than P1-glued flat quartz. We infer that increased surface roughness enhanced adhesion due to the increased effective contact areas of the substrates.

After the lap joint shear strength tests, all of the fracture surfaces of the samples in Figure 3 were within the azopolymer layer; i.e., the failure was within the polymer layer. Thus, the adhesion strengths were on the same order of magnitude for different substrates. We made drop-cast films of P1 on a glass substrate and a Teflon substrate. We tried to separate P1/glass and $\mathrm{P} 1 /$ Teflon. The failure was always within the P1 layer. These experiments demonstrated again that the interaction within the P1 layer was weaker than those between P1 and the substrates. These results coincide with the results in Figure 3c: When different substrates glued with P1 were separated, the failure was always within the P1 layer. When two substrates are glued using $\mathrm{P} 1$, the adhesion is switchable because photoinduced reversible solid-to-liquid transitions of P1 change the intrinsic interactions of the azopolymer chains. The abovementioned results demonstrate that P1 is a novel photoswitchable adhesive for different substrates.

We have demonstrated photocontrolled dry adhesion using P1. The next question is whether we can control underwater adhesion with light. We studied photocontrolled underwater adhesion using P1-glued quartz. Trans P1 firmly bonded quartz substrates and consequently lifted a copper block $(2.6 \mathrm{~kg})$ in water (Figure 4a). UV-light-induced trans-to-cis isomerization dramatically reduced adhesion. As a result, the copper block fell after UV irradiation within a minute (Movie S2). Switching underwater adhesion was achieved.

We quantified the underwater adhesion using tensile tests (Figure $4 \mathrm{~b}$ ). The underwater adhesion of trans-P1-glued quartz was $\sim 0.93 \mathrm{MPa}$. UV irradiation reduced the underwater adhesion to $\sim 0.08 \mathrm{MPa}$. We further investigated the reversibility of underwater adhesion under light-assisted and solvent-assisted processes (see more details in the Supporting Information). For the light-assisted process, two separated, wet quartz substrates with P1 adhesives were irradiated with UV 
(a)

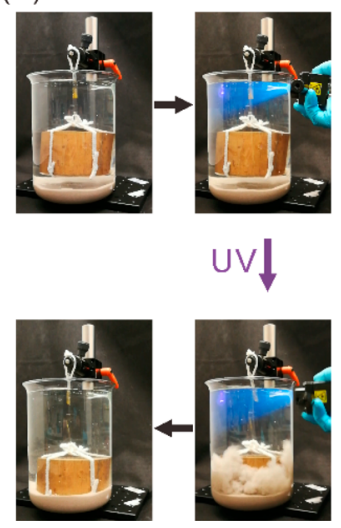

b)

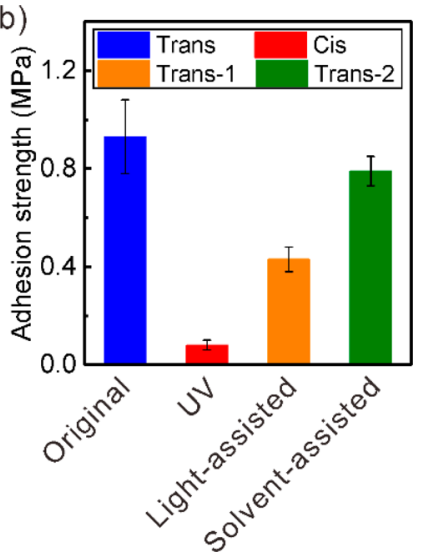

Figure 4. (a) Snapshots showing photocontrolled underwater adhesion (also see Movie S2). P1-glued quartz substrates lifted a copper block $(2.6 \mathrm{~kg})$ in water. The copper block fell after UV irradiation. (b) Underwater adhesion of trans-P1-glued substrates before irradiation (blue), after UV irradiation (red), after reuse with a light-assisted process (orange), and after reuse with a solvent-assisted process (green).

light $\left(365 \mathrm{~nm}, 71 \mathrm{~mW} / \mathrm{cm}^{2}, 15 \mathrm{~min}\right)$. The substrates with liquefied P1 were then clamped. Subsequent exposure to visible light $\left(530 \mathrm{~nm}, 5 \mathrm{~mW} / \mathrm{cm}^{2}, 15 \mathrm{~min}\right)$ solidified $\mathrm{P} 1$ and regenerated trans-P1-glued substrates, which had $\sim 46 \%$ of the original adhesion strength. The partial recovery may be due to the fact that water on the surface hinders the contact of azopolymer chains. We also tested reversibility via a solventassisted process. Two separated substrates were dried in an oven at $40{ }^{\circ} \mathrm{C}$ for $24 \mathrm{~h}$. Then, dichloromethane $(10 \mu \mathrm{L})$ was added to the cracked $\mathrm{P} 1$ on the substrates. The substrates were clamped and placed under a load $(50 \mathrm{~g})$ in a fume hood for 24 $\mathrm{h}$ before the underwater adhesion measurement. The underwater adhesion regenerated via the solvent-assisted process was $\sim 85 \%$ of the original process. For the solvent-assisted process, P1 was dissolved in dichloromethane, which formed a solution with much lower viscosity than that of cis $\mathrm{P} 1$. As a result, the surfaces could be wetted by the solution much better and the substrates were glued together with less imperfection. Therefore, the solvent-assisted process showed better recovery performance as compared to that of the light-assisted process.

In conclusion, azopolymer P1 is a switchable adhesive that can bond, debond, and rebond different substrates on demand. We also demonstrated the control of underwater adhesion using P1. The switchable adhesion is attributed to the novel photoinduced reversible solid-to-liquid transitions of P1. In contrast to other reversible adhesives, P1 does not require surface treatment of the substrates, allowing for a simple and efficient route to reversibly bond and separate surfaces. Another advantage of our method is that light has a high spatiotemporal resolution and can precisely control adhesion without contact. Our work also shows that reversible solid-toliquid transitions could be a general strategy for designing switchable adhesives. We expect that photoinduced reversible solid-to-liquid transitions will further improve materials recycling and automated production processes that require reversible bonding and debonding.

\section{ASSOCIATED CONTENT}

\section{Supporting Information}

The Supporting Information is available free of charge on the ACS Publications website at DOI: 10.1021/acsmacrolett.9b00459.

Materials, characterization, synthesis of the azopolymer $\mathrm{P} 1$, photoisomerization of $\mathrm{P} 1$ in a thin film, and AFM and optical microscopy images (PDF)

Light-controlled adhesion. Trans-P1-glued substrates lifting a bottle of water ( $500 \mathrm{~g})$. UV-light-induced trans-to-cis isomerization and reduced adhesion (MP4) Light-controlled underwater adhesion. Trans-P1-glued substrates lifting a copper block $(\sim 2.6 \mathrm{~kg})$. UV-lightinduced trans-to-cis isomerization and reduced adhesion (MP4)

\section{AUTHOR INFORMATION}

\section{Corresponding Authors}

*E-mail: siwu@ustc.edu.cn.

*E-mail: kongjie@nwpu.edu.cn.

\section{ORCID}

Kaloian Koynov: 0000-0002-4062-8834

Hans-Jürgen Butt: 0000-0001-5391-2618

Jie Kong: 0000-0002-9405-3204

Si Wu: 0000-0001-8156-346X

\section{Notes}

The authors declare no competing financial interest.

\section{ACKNOWLEDGMENTS}

The authors thank A. Hanewald for his technical support. This work was supported by the Deutsche Forschungsgemeinschaft (DFG, WU 787/2-1 and WU 787/8-1), the Thousand Talents Plan, the Fonds der Chemischen Industrie (FCI, No. 661548), the Natural Science Basic Research Plan in Shaanxi Province of China (2018JC-008, Distinguished Young Scholar), the Shanxi Scholarship Council of China (No. 201701D221029), and the Shanxi Province Hundred Talents Project.

\section{REFERENCES}

(1) Rodriguez-Emmenegger, C.; Preuss, C. M.; Yameen, B.; PopGeorgievski, O.; Bachmann, M.; Mueller, J. O.; Bruns, M.; Goldmann, A. S.; Bastmeyer, M.; Barner-Kowollik, C. Controlled Cell Adhesion on Poly(dopamine) Interfaces Photopatterned with Non-Fouling Brushes. Adv. Mater. 2013, 25, 6123-6127.

(2) Farrukh, A.; Paez, J. I.; Salierno, M.; del Campo, A. Bioconjugating Thiols to Poly(acrylamide) Gels for Cell Culture Using Methylsulfonyl Co-monomers. Angew. Chem., Int. Ed. 2016, 55, 2092-2096.

(3) Roling, O.; Stricker, L.; Voskuhl, J.; Lamping, S.; Ravoo, B. J. Supramolecular surface adhesion mediated by azobenzene polymer brushes. Chem. Commun. 2016, 52, 1964-1966.

(4) Li, W.; Xu, J.; Li, X.; Li, J.; Li, X.; Li, B.; Wang, Y.; Wu, L. Wet and Functional Adhesives from One-Step Aqueous Self-Assembly of Natural Amino Acids and Polyoxometalates. Angew. Chem., Int. Ed. 2017, 56, 8731-8735.

(5) Li, X.; Du, Z.; Song, Z.; Li, B.; Wu, L.; Liu, Q.; Zhang, H.; Li, W. Bringing hetero- polyacid- based underwater adhesive as printable cathode coating for self- powered electrochromic aqueous batteries. Adv. Funct. Mater. 2018, 28, 1800599.

(6) Lee, B. P.; Messersmith, P. B.; Israelachvili, J. N.; Waite, J. H. Mussel- inspired adhesives and coatings. Annu. Rev. Mater. Res. 2011, 41, 99-132. 
(7) Li, L.; Yan, B.; Yang, J.; Chen, L.; Zeng, H. Novel musselinspired injectable self-healing hydrogel with anti-biofouling property. Adv. Mater. 2015, 27, 1294-1299.

(8) Heinzmann, C.; Coulibaly, S.; Roulin, A.; Fiore, G. L.; Weder, C. Light-Induced Bonding and Debonding with Supramolecular Adhesives. ACS Appl. Mater. Interfaces 2014, 6, 4713-4719.

(9) Faghihnejad, A.; Feldman, K. E.; Yu, J.; Tirrell, M. V.; Israelachvili, J. N.; Hawker, C. J.; Kramer, E. J.; Zeng, H. Adhesion and Surface Interactions of a Self-Healing Polymer with Multiple Hydrogen-Bonding Groups. Adv. Funct. Mater. 2014, 24, 2322-2333.

(10) Ju, G.; Cheng, M.; Guo, F.; Zhang, Q.; Shi, F. ElasticityDependent Fast Underwater Adhesion Demonstrated by Macroscopic Supramolecular Assembly. Angew. Chem., Int. Ed. 2018, 57, 89638967.

(11) Ju, G.; Guo, F.; Zhang, Q.; Kuehne, A. J. C.; Cui, S.; Cheng, M.; Shi, F. Self-Correction Strategy for Precise, Massive, and Parallel Macroscopic Supramolecular Assembly. Adv. Mater. 2017, 29, 1702444.

(12) Liu, M.; Liu, P.; Lu, G.; Xu, Z.; Yao, X. Multiphase-Assembly of Siloxane Oligomers with Improved Mechanical Strength and WaterEnhanced Healing. Angew. Chem., Int. Ed. 2018, 57, 11242-11246.

(13) Wang, D.; Ha, Y.; Gu, J.; Li, Q.; Zhang, L.; Yang, P. 2D Protein Supramolecular Nanofilm with Exceptionally Large Area and Emergent Functions. Adv. Mater. 2016, 28, 7414-7423.

(14) Guo, J.; Yuan, C.; Guo, M.; Wang, L.; Yan, F. Flexible and voltage-switchable polymer velcro constructed using host-guest recognition between poly(ionic liquid) strips. Chem. Sci. 2014, 5, 3261

(15) Ahn, Y.; Jang, Y.; Selvapalam, N.; Yun, G.; Kim, K. Supramolecular Velcro for Reversible Underwater Adhesion. Angew. Chem., Int. Ed. 2013, 52, 3140-3144.

(16) Yamaguchi, H.; Kobayashi, Y.; Kobayashi, R.; Takashima, Y.; Hashidzume, A.; Harada, A. Photoswitchable gel assembly based on molecular recognition. Nat. Commun. 2012, 3, 603.

(17) Saito, S.; Nobusue, S.; Tsuzaka, E.; Yuan, C.; Mori, C.; Hara, M.; Seki, T.; Camacho, C.; Irle, S.; Yamaguchi, S. Light-melt adhesive based on dynamic carbon frameworks in a columnar liquid-crystal phase. Nat. Commun. 2016, 7, 12094.

(18) Akiyama, H.; Fukata, T.; Yamashita, A.; Yoshida, M.; Kihara, H. Reworkable adhesives composed of photoresponsive azobenzene polymer for glass substrates. J. Adhes. 2017, 93, 823-830.

(19) Akiyama, H.; Yoshida, M. Photochemically Reversible Liquefaction and Solidification of Single Compounds Based on a Sugar Alcohol Scaffold with Multi Azo-Arms. Adv. Mater. 2012, 24, 2353-2356.

(20) Ito, S.; Yamashita, A.; Akiyama, H.; Kihara, H.; Yoshida, M. Azobenzene-Based (Meth)acrylates: Controlled Radical Polymerization, Photoresponsive Solid-Liquid Phase Transition Behavior, and Application to Reworkable Adhesives. Macromolecules 2018, 51, $3243-3253$.

(21) Schenzel, A. M.; Klein, C.; Rist, K.; Moszner, N.; BarnerKowollik, C. Reversing Adhesion: A Triggered Release Self-Reporting Adhesive. Adv. Sci. 2016, 3, 1500361.

(22) Ye, Z.; Lum, G. Z.; Song, S.; Rich, S.; Sitti, M. Phase Change of Gallium Enables Highly Reversible and Switchable Adhesion. Adv. Mater. 2016, 28, 5088-5092.

(23) Chen, C.-M.; Chiang, C.-L.; Lai, C.-L.; Xie, T.; Yang, S. Buckling-Based Strong Dry Adhesives Via Interlocking. Adv. Funct. Mater. 2013, 23, 3813-3823.

(24) Drotlef, D. M.; Blumler, P.; del Campo, A. Magnetically Actuated Patterns for Bioinspired Reversible Adhesion (Dry and Wet). Adv. Mater. 2014, 26, 775-779.

(25) Gelebart, A. H.; Liu, D.; Mulder, D. J.; Leunissen, K. H. J.; van Gerven, J.; Schenning, A. P. H. J.; Broer, D. J. Photoresponsive Sponge-Like Coating for On-Demand Liquid Release. Adv. Funct. Mater. 2018, 28, 1705942.

(26) Liu, D.; Broer, D. J. Self-assembled Dynamic 3D Fingerprints in Liquid-Crystal Coatings Towards Controllable Friction and Adhesion. Angew. Chem., Int. Ed. 2014, 53, 4542-4546.
(27) Akiyama, H.; Kanazawa, S.; Okuyama, Y.; Yoshida, M.; Kihara, H.; Nagai, H.; Norikane, Y.; Azumi, R. Photochemically Reversible Liquefaction And Solidification Of Multiazobenzene Sugar-Alcohol Derivatives And Application To Reworkable Adhesives. ACS Appl. Mater. Interfaces 2014, 6, 7933-7941.

(28) Weis, P.; Tian, W.; Wu, S. Photoinduced Liquefaction of Azobenzene-Containing Polymers. Chem. - Eur. J. 2018, 24, 64946505.

(29) Zhou, H.; Xue, C.; Weis, P.; Suzuki, Y.; Huang, S.; Koynov, K.; Auernhammer, G. K.; Berger, R.; Butt, H.-J.; Wu, S. Photoswitching of glass transition temperatures of azobenzene-containing polymers induces reversible solid-to-liquid transitions. Nat. Chem. 2017, 9, $145-151$.

(30) Uchida, E.; Azumi, R.; Norikane, Y. Light-induced crawling of crystals on a glass surface. Nat. Commun. 2015, 6, 7310.

(31) Yue, Y.; Norikane, Y.; Azumi, R.; Koyama, E. Light-induced mechanical response in crosslinked liquid-crystalline polymers with photoswitchable glass transition temperatures. Nat. Commun. 2018, 9, 3234. 